\title{
Burstein Moss effect in nanocrystalline CaS : Ce
}

\author{
GEETA SHARMA*, PUJA CHAWLA, S P LOCHAB ${ }^{\dagger}$ and NAFA SINGH \\ Department of Physics, Kurukshetra University, Kurukshetra 136 119, India \\ ${ }^{\dagger}$ Inter University Accelerator Centre, Aruna Asaf Ali Marg, New Delhi 110 067, India
}

MS received 27 July 2009; revised 16 September 2009

\begin{abstract}
The nanocrystalline CaS : Ce nanophosphors are synthesized by wet chemical co-precipitation method. The particles possess an average size of $10 \mathrm{~nm}$ as calculated using Debye-Scherrer formula. The particle size and the crystalline nature of the formed nanoparticles are confirmed by TEM micrograph. The optical studies are carried out using UV-Vis absorption spectroscopy. The absorption edge is found to show blue shift with increasing cerium concentration. The shift may be attributed to Burstein Moss effect.
\end{abstract}

Keywords. Burstein Moss effect; calcium sulphide; nanophosphors.

\section{Introduction}

Calcium sulphide (CaS) is being investigated for long time because of its interesting properties such as long afterglow, high accumulation capability and high sensitivity to infrared radiations. Different properties of calcium sulphide have been intensively investigated both experimentally and theoretically. Calcium sulphide belongs to II-VI group and crystallizes into $\mathrm{NaCl}$ type structure. It is a well known material for its luminescent behaviour. Also known as Lenard phosphor, it is the most versatile material for various applications. CaS is a good phosphor for cathode ray tubes (Marwaha et al 1980), TV screens, fluorescent lamps and thermoluminescent dosimeters (Marwaha et al 1979; Marwaha et al 1981). Alkaline earth sulphides are of great interest since impurities can be easily introduced because of their less ionic character than alkali halides (Han et al 1993). Being a wide bandgap semiconductor, $\mathrm{CaS}$ can accommodate large variety of dopants, and by just changing the host dopant combination one can tailor whole of the visible spectrum.

Advent of nanotechnology has stimulated interest in nanocrystalline form of bulk phosphors. The properties of nanophosphors are entirely different from their bulk counter parts, e.g. the bulk semiconductor become insulator when the dimensions of the particle is reduced to nanometer regime (Kumar et al 2006). Further, the nanophosphors become important for those applications where smaller particle size of the phosphor particle is required, e.g. for higher resolution in computer monitors one requires smaller phosphor particles (Van Haecke et al 2007). Small particles cause lower screen loading.

\footnotetext{
*Author for correspondence (g.sharma.ku@gmail.com)
}

The CaS nanophosphors were prepared chemically by a large number of scholors. Sun et al (2002) prepared CaS : $\mathrm{Eu}$ nanoparticles through wet chemical co-precipitation method and obtained monodispersed particles with narrow size distribution. Sun et al (2002) and Kumar et al (2006) prepared $\mathrm{CaS}: \mathrm{Bi}$ nanoparticles with the same method. Sawanda et al (2006) prepared CaS : Eu nanoparticles using alkoxide method. Wang et al (2002) prepared undoped CaS and SrS nanophosphors using solvothermal process.

In the present study we have prepared $\mathrm{CaS}$ : Ce nanophosphors using wet chemical co-precipitation method (Sun et al 2002) and studied their optical absorption characteristics with varying cerium concentrations.

\section{Experimental}

The CaS:Ce nanophosphors were prepared by wet chemical co-precipitation method. All the chemicals used for the synthesis were of analytical grade. $\mathrm{CaCl}_{2} \cdot 5 \mathrm{H}_{2} \mathrm{O}$, $\mathrm{Na}_{2} \mathrm{~S} \cdot 9 \mathrm{H}_{2} \mathrm{O}$ and $\mathrm{Ce}\left(\mathrm{NO}_{3}\right)_{3} \cdot 6 \mathrm{H}_{2} \mathrm{O}$ were the starting materials. Ethanol was used as the solvent for dissolving the starting materials, since the polarity of ethanol was perfect for dissolving original reagents. 1-Thioglycerol was used as the capping agent. A $0.0125 \mathrm{M}$ sulphide solution was prepared by dissolving $0.6 \mathrm{~g} \mathrm{Na} 2 \mathrm{~S}_{2} \cdot 9 \mathrm{H}_{2} \mathrm{O}$ and $0.21 \mathrm{ml}$ of 1-thioglycerol in $200 \mathrm{ml}$ of ethanol. $0.025 \mathrm{M}$ solution of $\mathrm{CaCl}_{2} \cdot 2 \mathrm{H}_{2} \mathrm{O}$ was prepared by dissolving $0.7351 \mathrm{~g}$ of $\mathrm{CaCl}_{2} \cdot 2 \mathrm{H}_{2} \mathrm{O}$ in $200 \mathrm{ml}$ in ethanol and $0.01 \mathrm{M}$ solution of cerium was prepared by dissolving $0.1736 \mathrm{~g}$ of $\mathrm{Ce}\left(\mathrm{NO}_{3}\right)_{3} \cdot 6 \mathrm{H}_{2} \mathrm{O}$ in $40 \mathrm{ml}$ of ethanol.

For the synthesis of $\mathrm{CaS}$ : Ce nanoparticles, $200 \mathrm{ml}$ of $\mathrm{Na}_{2} \mathrm{~S} \cdot 9 \mathrm{H}_{2} \mathrm{O}$ and 1-thoiglycerol was sonicated for $30 \mathrm{~min}$. To the $200 \mathrm{ml}$ solution of $\mathrm{CaCl}_{2}, 40 \mathrm{ml}$ of cerium nitrate solution was added and $\mathrm{N}_{2}$ was continuously bubbled into the reaction mixture to avoid formation of calcium car- 
bonate. The solution was continuously deoxygenated with $\mathrm{N}_{2}$ for $15 \mathrm{~min}$ followed by the addition of $200 \mathrm{ml}$ of sulphide solution. Then the mixture was stirred for $3 \mathrm{~h}$ continuously using a magnetic stirrer. Particle growth took place at this stage via Oswald ripening. After this, solution was evaporated till the initial $440 \mathrm{ml}$ solution became approximately $100 \mathrm{ml}$. Tetrahydrofuran (THF) was added dropwise till the solution became turbid. The remaining solution was again stirred for $2 \mathrm{~h}$. The nanoparticles so formed were collected through centrifugation. The precipitates formed were washed with ethanol twothree times and then dried in vacuum at $70^{\circ} \mathrm{C}$ for $4 \mathrm{~h}$. These dried precipitates were then collected in dry sample tubes for further study.

The size and the shape of the product were determined by transmission electron microscopy (TEM) and X-ray diffraction spectroscopy (XRD). TEM was carried out on a H-7500 (Hitachi Ltd. Tokyo Japan) operated at $120 \mathrm{kV}$. The phase purity of the samples is checked out by X-ray diffraction technique using $\mathrm{CuK}_{\alpha}$ radiation from D8 advance model of Bruker (Germany). The optical studies were done UV-Vis absorption spectroscopy. The optical absorption spectra were recorded on an UV-Vis 2500 PC spectrophotometer.

\section{Results and discussion}

The phase purity of the formed nanoparticles was checked using X-ray diffraction. Figure 1 shows the XRD pattern for $\mathrm{CaS}: \mathrm{Ce}(0.02 \mathrm{M})$ nanoparticles. The average crystallite size of the formed nanoparticles was estimated from the FWHM of the most intense XRD peak using DebyeScherrer formula (Cullity 1959)

$$
D=0.89 \lambda / \beta \cos \theta,
$$

where $D$ is the average crystallite size of the nanoparticles, $\lambda$ is the wavelength of $\mathrm{CuK}_{\alpha}(0 \cdot 154 \mathrm{~nm})$ radiation, $\beta$

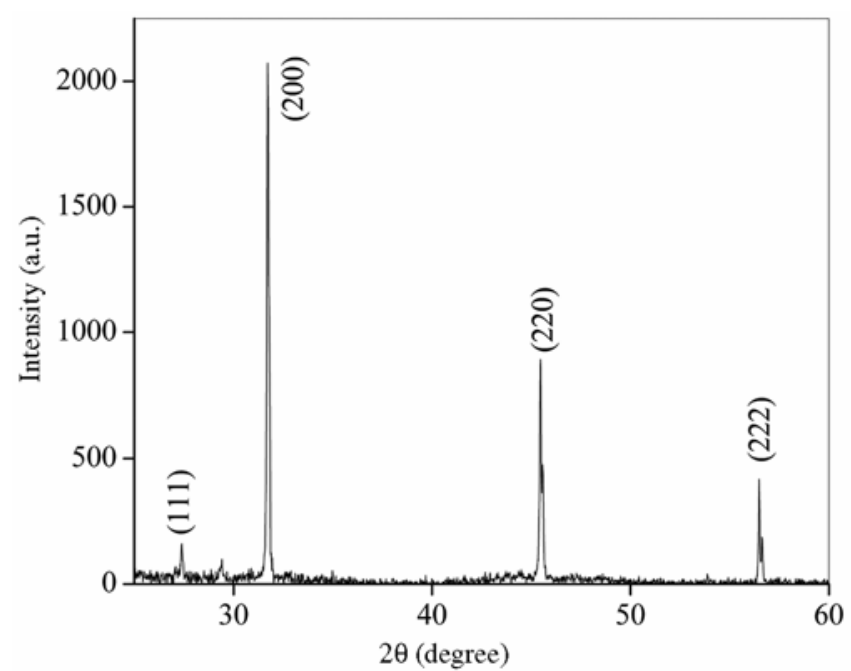

Figure 1. XRD pattern for $\mathrm{CaS}: \mathrm{Ce}(0.02 \mathrm{M})$ nanoparticles. in radians is the full width at half maxima (FWHM) of the XRD peak and $\theta$ is the Bragg angle. The average crystallite size of the particles calculated using formula (1) comes out to be $\sim 10 \mathrm{~nm}$. The obtained XRD pattern is in good agreement with the standard data available in JCPDS Card number 77-2011 and shows cubic crystalline phase of the host lattice. Figure 2 shows the XRD spectra for different concentrations of cerium. It is observed from figure 2 that doping does not affect the peak width which indicates that average particle size (calculated using Debye-Scherrer formula) remain almost unchanged with different cerium concentrations. There is slight variation in the peak position and lattice constant decreases with increasing cerium doping. This indicates the effective incorporation of cerium. Table 1 summarizes the values of diffraction angle $(2 \theta)$, crystallite size and lattice constant for different cerium concentration.

Figure 3 shows the TEM image of as prepared CaS : Ce $(0.02 \mathrm{M})$ nanoparticles. The image reveals an average diameter range of these nanoparticles from 10-20 nm which is in agreement with the particle size obtained from XRD pattern. The particles possess a spherical shape and are well dispersed with no agglomeration in them. Figure 3 (inset) shows the selected area electron diffraction pattern for $\mathrm{CaS}: \mathrm{Ce}$ nanoparticles which reveals the crystalline $\mathrm{CaS}$ structure.

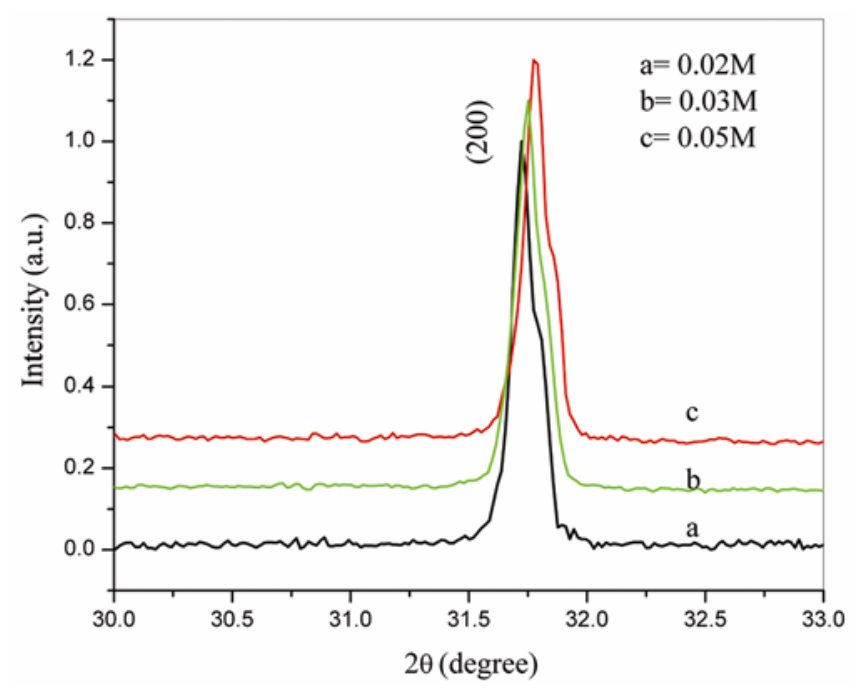

Figure 2. XRD pattern for $\mathrm{CaS}$ : Ce nanoparticles for different concentrations of cerium

Table 1. Calculated crystallite size of Ce doped CaS nanoparticles for different concentration of cerium.

\begin{tabular}{lccc}
\hline $\begin{array}{l}\text { Concentration } \\
\text { of cerium }\end{array}$ & $2 \theta^{\circ}$ & $\begin{array}{l}\text { Crystallite } \\
\text { size }(\mathrm{nm})\end{array}$ & $\begin{array}{c}\text { Lattice } \\
\text { constant }(\AA)\end{array}$ \\
\hline $0.02 \mathrm{M}$ & 31.725 & 9.96 & 5.636 \\
$0.03 \mathrm{M}$ & 31.749 & 9.67 & 5.632 \\
$0.05 \mathrm{M}$ & 31.782 & 12.10 & 5.626 \\
\hline
\end{tabular}


Absorption of light by the semiconductor nanoparticles depend on the gap between the conduction and valence band, and the impurity concentration in the host lattice. Figure 4 shows the absorption spectra of $\mathrm{CaS}: \mathrm{Ce}$ nanoparticles with varying cerium concentrations. From figure 4 it is clear that as the impurity concentration increases there is shift in the absorption edge towards

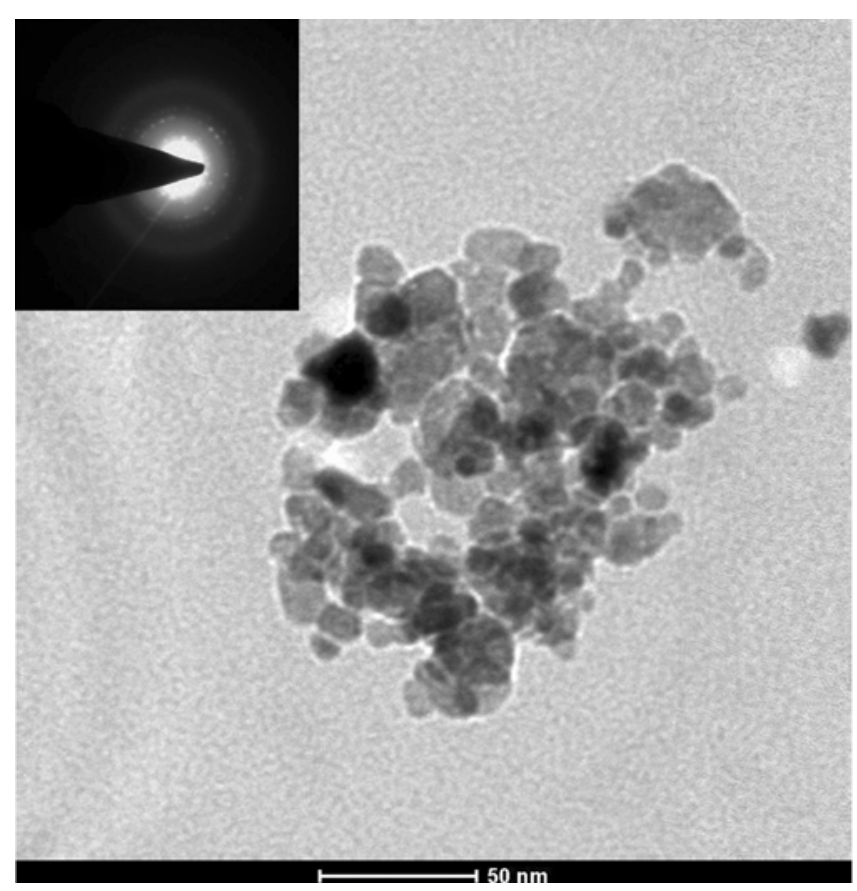

Figure 3. TEM image of $\mathrm{CaS}: \mathrm{Ce}(0.02 \mathrm{M})$ nanoparticles.

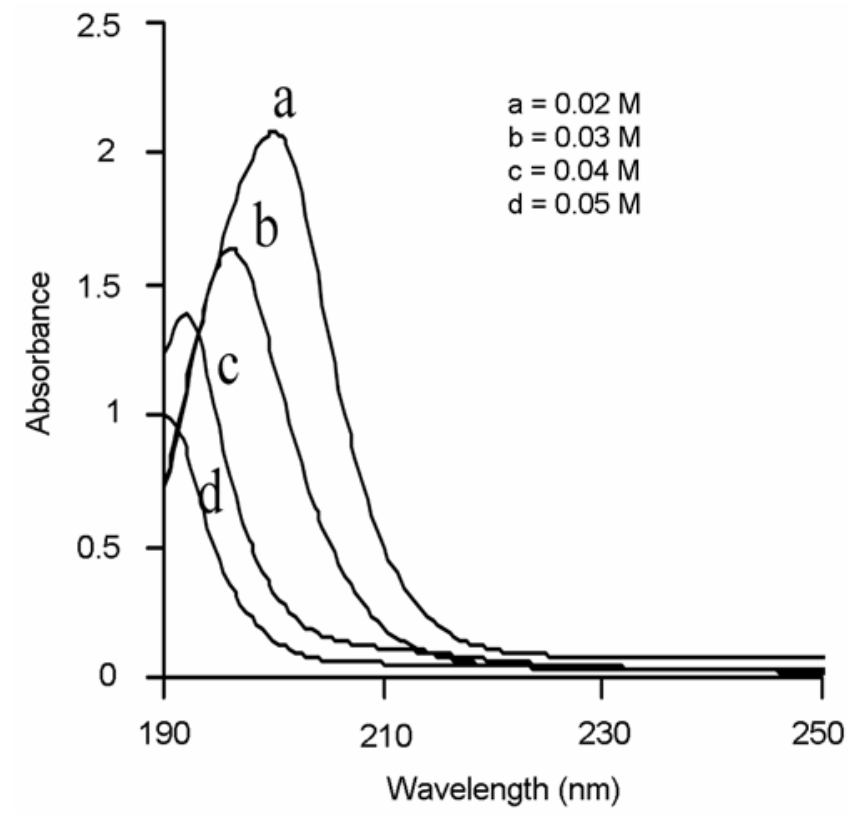

Figure 4. Optical absorption spectra of $\mathrm{CaS}$ : Ce with increasing cerium concentration. higher energy. The possible reason for the blue shift may be Burstein Moss effect (Kim et al 1992). With increase in the amount of impurity added, excess carriers are supplied by the impurity leading to blue shift in the absorption spectrum (Alexei et al 2005; Shen et al 2005; Zaera et al 2008). This shift is known as Burstein Moss shift. The Burstein Moss shift is related to concentration of impurity in the material and radiation intensity. Some authors have reported the photoconductive behaviour of cerium-doped hosts with varying cerium concentrations and has found that photocurrent rises with increasing cerium concentration which shows that increasing cerium doping increases the charge carriers in the conduction band (Choi et al 1999; Jia et al 2002). When a semiconductor nanoparticle is photo excited, large numbers of charge carriers are photo generated and these photo-generated electrons occupy states at the bottom of conduction band. After the thermal relaxation of these charge carriers to the lower viberational levels in the conduction band,

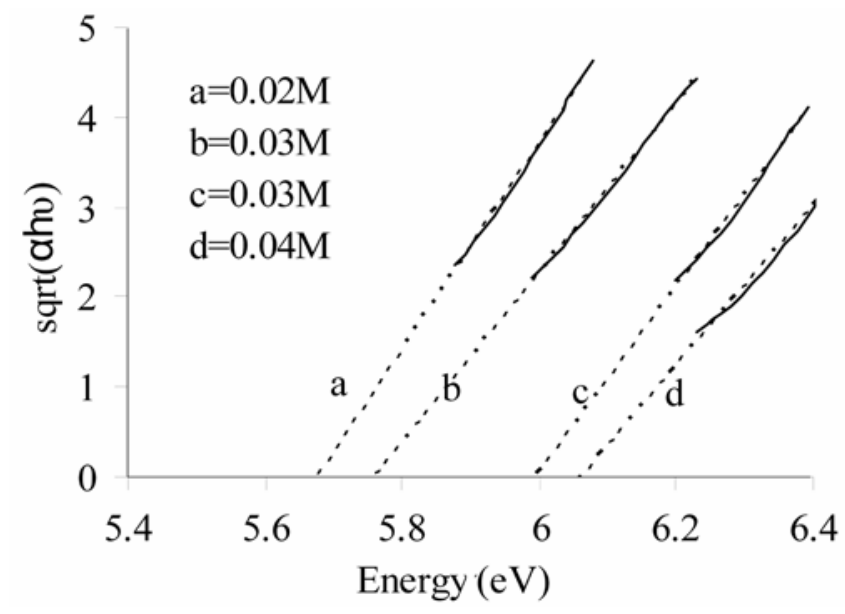

Figure 5. Band gap of $\mathrm{CaS}: \mathrm{Ce}$ nanoparticles with varying cerium concentration.

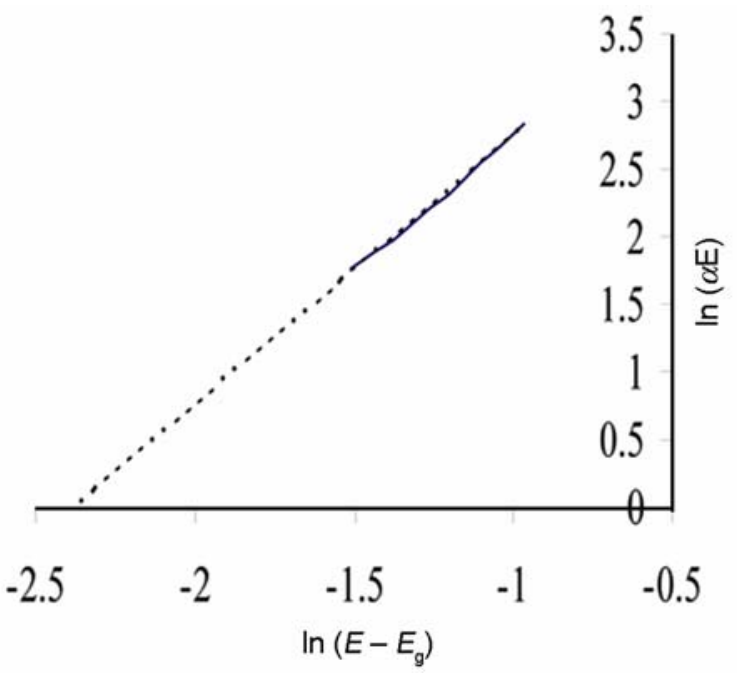

Figure 6. Plot against the coordinates $\ln (\alpha E)$ and $\ln \left(E-E_{\mathrm{g}}\right)$. 
there is a considerable population in the lower viberational levels of the conduction band, i.e. the lower viberational levels are well populated. Now the photo excitation of such a particle requires $E_{\mathrm{g}}+\Delta E_{\mathrm{b}}$ energy, where in $\Delta E_{\mathrm{b}}$ is the excess energy needed to transfer the electrons to the unoccupied electronic sublevels closest to the bottom of the conduction band (Stroyuk et al 2005). This shift in $\Delta E_{\mathrm{b}}$ is called the Burstein Moss shift. The blue shift in the absorption edge also supports the fact that cerium acts as donor in CaS host as stated by earlier researchers.

Figure 5 shows a plot of $\sqrt{ } \alpha h v$ vs $h v$ for varying concentrations of cerium. The optical band gap is calculated using formula (Pankove 1971; Sze 2004)

$$
(\alpha h v)^{1 / n} \sim\left(h v-E_{\mathrm{g}}\right),
$$

where $\alpha$ is the absorption coefficient, $h v$ is the energy of the absorbed photon and $n$ is an integer whose value depends on the nature of transition. Value of $n$ is $1 / 2,2$, $3 / 2$ and 3 for allowed direct transition, allowed indirect transition, forbidden direct transition and forbidden indirect transition, respectively.

Band gap is calculated by extrapolating the $\sqrt{ } \alpha h v$ vs $h v$, taking the point where $\sqrt{ } \alpha h v$ approaches zero. The band gap shows an increase with dopant concentration from $0.02 \mathrm{M}$ to $0.05 \mathrm{M}$. The $\mathrm{CaS}:$ Ce possesses indirect band gap. The band gap changes with doping concentration from $5.67 \mathrm{eV}$ for $0.02 \mathrm{M}$ to $6.06 \mathrm{eV}$ for $0.05 \mathrm{M}$ of cerium. Further, the value of $n$ can be obtained as the tangent of relationship (2), linearized against the coordinates $\ln (\alpha h v)$ and $\ln \left(h v-E_{\mathrm{g}}\right)$ as shown in figure 6 (Stroyuk et al 2005). The value of $n$ comes out to be $1.99 \pm 0.03$ corresponding to allowed indirect transition.

\section{Conclusion}

We have synthesized CaS nanocrystalline phosphor activated by $\mathrm{Ce}^{3+}$ ions. These are characterized by XRD and TEM spectroscopy. The optical absorption studies in CaS : Ce nanocrystalline phosphors have been presented. The shift in absorption edge towards lower wavelength side, with increasing cerium concentration may be attributed to Burstein Moss effect.

\section{Acknowledgement}

One of the authors GS is thankful to IUAC, New Delhi for financial help in the form of a fellowship under the project UFUP-44305.

\section{References}

Alexei E V, Yutaka F, Zhang X T, Jin M, Taketoshi M and Akira F 2005 J. Phys. Chem. B109 24441

Choi J, Basun S A, Lu L, Yen W M and Happek U 1999 J. Lumin. 83461

Cullity B D 1959 Elements of X-ray diffraction (AddisonWesley: London)

Han M, Oh S -J, Park J H and Park H L 1993 J. Appl. Phys. 73 4546

Jia D, Meltzer R S and Yen W M 2002 J. Lumin. 991

Kim D T, Yu K S, Kim W T, Kim C D and Park H L $1992 J$. Mater. Sci. Lett. 11886

Kumar V, Kumar R, Lochab S P and Singh N 2006 J. Phys.: Condens. Matter. 185029

Marwaha G L, Singh N and Mathur V K 1979 Mater. Res. Bull. 141489

Marwaha G L, Singh N and Mathur V K 1980 Rad. Effects 53 25

Marwaha G L, Singh N, Nagpal J S and Mathur V K 1981 Rad. Effects $\mathbf{5 5} 85$

Pankove J J 1971 Optical process in semiconductors (New York: Dover)

Sawanda N, Chen T and Isobe T 2006 J. Alloy Compd. 824 408

Shen G Z, Cho J H, Yoo J K, Yi G C and Lee C J 2005 J. Phys. Chem. B109 5491

Stroyuk A L, Kryukov A I, Kuchmii Ya S and Pokhodenko V D 2005 Theoretical and Experimental Chem. 412

Sun B Q, Yi G S, Yang F G, Chen D P, Zhou Y X and Cheng J 2002 J. Mater. Chem. 121194

Sze S M 2004 Physics of semiconductor devices (New York: Wiley)

Van Haecke J E, Smet P F, De Keyser K and Poelman D 2007 J. Electrochem. Soc. $\mathbf{1 5 4} 278$

Wang C et al 2002 Chem. Phys. Lett. 351385

Zaera R T, Mora-Seró I and Bisquert J 2008 J. Phys. Chem. C112 16318 